\title{
Diagrammatic Monte Carlo study of the Fermi polaron in two dimensions
}

\author{
Jonas Vlietinck, ${ }^{1}$ Jan Ryckebusch, ${ }^{1}$ and Kris Van Houcke ${ }^{1,2}$ \\ ${ }^{1}$ Department of Physics and Astronomy, Ghent University, Proeftuinstraat 86, 9000 Gent, Belgium \\ ${ }^{2}$ Laboratoire de Physique Statistique, Ecole Normale Supérieure, UPMC, Université Paris Diderot, \\ CNRS, 24 rue Lhomond, 75231 Paris Cedex 5, France
}

(Received 4 December 2013; revised manuscript received 30 January 2014; published 19 February 2014)

\begin{abstract}
We study the properties of the two-dimensional Fermi polaron model in which an impurity attractively interacts with a Fermi sea of particles in the zero-range limit. We use a diagrammatic Monte Carlo (DiagMC) method which allows us to sample a Feynman diagrammatic series to very high order. The convergence properties of the series and the role of multiple particle-hole excitations are discussed. We study the polaron and molecule energy as a function of the coupling strength, revealing a transition from a polaron to a molecule in the ground state. We find a value for the critical interaction strength which complies with the experimentally measured one and predictions from variational methods. For all considered interaction strengths, the polaron $Z$ factor from the full diagrammatic series almost coincides with the one-particle-hole result. We also formally link the DiagMC and the variational approaches for the polaron problem at hand.
\end{abstract}

DOI: 10.1103/PhysRevB.89.085119

PACS number(s): 05.30.Fk, 03.75.Ss, 02.70.Ss

\section{INTRODUCTION}

Experiments with ultracold gases are a powerful tool to investigate the (thermo)dynamics of quantum many-body systems under controlled circumstances. With Feshbach resonances [1], for example, one has the ability to tune the interaction strength. Optical potentials [2] can be exploited to modify the dimensionality of the studied systems. The properties of a single impurity that interacts strongly with a background gas, for example, can be addressed with ultracold atoms.

The so-called Fermi polaron problem refers to a single spin-down impurity that is coupled to a noninteracting spin-up Fermi sea (FS). This problem corresponds to the extreme limit of spin imbalance in a two-component Fermi gas [3-5] and has implications on the phase diagram of the strongly spin-polarized Fermi gas [6-8]. At weak attraction, one expects a "polaron" state [9], in which the impurity is dressed with density fluctuations of the spin-up Fermi gas. Recent experiments have observed indications of a transition from this polaronic state to a molecular state (a two-body bound state of the impurity and an atom of the sea) upon increasing the attraction strength in three dimensions (3D) [10] and in two dimensions (2D) [11]. Experimentally, the $2 \mathrm{D}$ regime can be accomplished by means of a transverse trapping potential $V(z)=\frac{1}{2} m \omega_{z}^{2} z^{2}$ (here, $\omega_{z}$ is the frequency and $z$ is the transverse direction) that fulfills the condition $k_{B} T \ll \epsilon_{F} \ll \hbar \omega_{z}$ ( $T$ is the temperature and $\epsilon_{F}$ is the Fermi energy of the FS). When excitations in the $z$ dimension are possible, one reaches the so-called quasi-2D regime $[12,13]$. The purely $2 \mathrm{D}$ limit is reached for $\epsilon_{F} / \hbar \omega_{z} \rightarrow 0$ and will be the subject of this paper.

The existence of a polaron-molecule transition in 3D has been predicted with the aid of the diagrammatic Monte Carlo (DiagMC) method [14-16] and of variational methods [9,17-19]. For the latter, the maximum number of particle-hole (p-h) excitations of the FS is limited to one or two [9,17-19]. One might naively expect that the role of quantum fluctuations increases in importance with decreasing dimensionality and that high-order $\mathrm{p}$-h excitations could become more important in one and two dimensions. For the one-dimensional (1D) Fermi polaron the known analytical solution displays no polaron-molecule transition [20]. Like for the 3D polaron, the approximate method in which the truncated Hilbert space contains one $\mathrm{p}-\mathrm{h}$ and two $\mathrm{p}$-h excitations of the FS gives results for the $1 \mathrm{D}$ polaron approaching the exact solution $[21,22]$. In 2D, the Fermi polaron properties have been studied with variational wave functions [23-25]. To observe a polaron-molecule transition in $2 \mathrm{D}$ it is crucial to include particle-hole excitations in both the polaron and molecule wave functions [24]. In the limit of weak interactions, the $1 \mathrm{p}-\mathrm{h}$ and $2 \mathrm{p}$-h variational Ansätze for the polaron branch provide similar results. Surprisingly, this is also the situation for strong correlations [25].

In this work we focus on the 2D Fermi polaron for attractive interactions and study the role of multiple particle-hole (mp-h) excitations for the ground-state properties of the system. The quasiparticle properties of the polaron are computed with the DiagMC method. This technique evaluates stochastically to high order a series of Feynman diagrams for the one-particle and two-particle self-energies. For the details of the DiagMC method and the adopted method for determining the groundstate energies from the computed self-energies, we refer to Refs. $[15,16]$. In this work we present DiagMC predictions for the interaction-strength dependence of the polaronic and molecular ground-state properties in 2D. We first briefly discuss the model and the diagrammatic method. We then discuss the results of the simulations, with particular emphasis on the role of the mp-h excitations. We also discuss how variational results for the polaron problem can be obtained within the DiagMC formalism.

\section{FORMALISM}

We consider a two-component Fermi gas confined to 2D at temperature $T=0$. Even though we will consider the zerorange interaction in continuous space, we start from a lattice model to avoid ultraviolet divergences from the onset. The 
corresponding Hamiltonian reads

$$
\begin{aligned}
\hat{H}= & \sum_{\mathbf{k} \in \mathcal{B}, \sigma=\uparrow \downarrow} \epsilon_{\mathbf{k} \sigma} \hat{c}_{\mathbf{k} \sigma}^{\dagger} \hat{c}_{\mathbf{k} \sigma} \\
& +g_{0} \sum_{\mathbf{r}} b^{2} \hat{\Psi}_{\uparrow}^{\dagger}(\mathbf{r}) \hat{\Psi}_{\downarrow}^{\dagger}(\mathbf{r}) \hat{\Psi}_{\downarrow}(\mathbf{r}) \hat{\Psi}_{\uparrow}(\mathbf{r}),
\end{aligned}
$$

with $\hat{\Psi}_{\sigma}(\mathbf{r})$ and $\hat{c}_{\mathbf{k}, \sigma}$ being the operators for annihilating a spin- $\sigma$ fermion with mass $m_{\sigma}$ and dispersion $\epsilon_{\mathbf{k} \sigma}=k^{2} / 2 m_{\sigma}$ in position and momentum space. The components of the position vector $\mathbf{r}$ are integer multiples of the finite lattice spacing $b$. Further, $g_{0}$ is the bare interaction strength. The wave vectors $\mathbf{k}$ are in the first Brillouin zone $\mathcal{B}=]-\pi / b, \pi / b]$. The continuum limit is reached for $b \rightarrow 0$. We adopt the convention $\hbar=1$ and consider the mass-balanced case $m_{\uparrow}=m_{\downarrow}=m$. We make use of the $T$ matrix [26] for a single spin- $\uparrow$ and spin- $\downarrow$ fermion in vacuum,

$$
-\frac{1}{g_{0}}=\frac{1}{\mathcal{V}} \sum_{\mathbf{k} \in \mathcal{B}} \frac{1}{\varepsilon_{B}+\epsilon_{\mathbf{k} \uparrow}+\epsilon_{\mathbf{k} \downarrow}},
$$

where $\mathcal{V}$ is the area of the system and $\varepsilon_{B}$ is the twobody binding energy [which depends on $m, g_{0}$, and $b$ and $\left.\varepsilon_{B}(m, g, b)>0\right]$ of a weakly bound state. Such a state always exists for an attractive interaction in 2D. With the above relation we eliminate the bare interaction strength $g_{0}$ in favor of the quantity $\varepsilon_{B}$. Moreover, the diagrammatic approach allows us to take the continuum limit $b \rightarrow 0$ and $g_{0} \rightarrow 0^{-}$ while keeping $\varepsilon_{B}$ fixed. Summing all ladder diagrams gives a partially dressed interaction vertex $\Gamma^{0}$ :

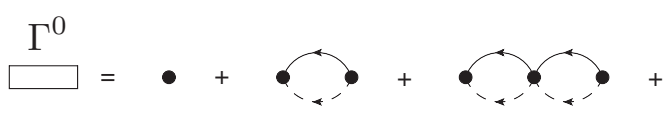

where the dot represents the bare interaction vertex $g_{0}$ and the lines represent bare-particle propagators for the spindown impurity (dashed lines) and the spin-up Fermi sea (solid lines). In momentum-imaginary frequency this graphical representation corresponds to

$$
\left[\Gamma^{0}(p, i \Omega)\right]^{-1}=g_{0}^{-1}-\Pi^{0}(p, i \Omega),
$$

with

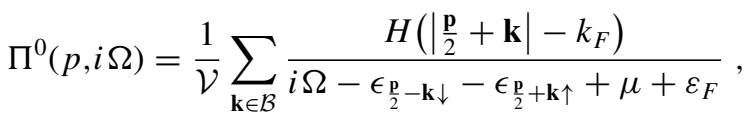

with $H(x)$ being the Heaviside step function and $\mu<0$ being a free parameter representing an energy offset of the impurity dispersion. Further, $k_{F}$ and $\varepsilon_{F}=\frac{k_{F}^{2}}{2 m}$ are the Fermi momentum and the Fermi energy of the spin-up sea. The combination of Eqs. (2) and (4) gives

$$
\begin{aligned}
\frac{1}{\Gamma^{0}(p, i \Omega)}= & -\frac{1}{\mathcal{V}} \sum_{\mathbf{k} \in \mathcal{B}}\left[\frac{1}{\varepsilon_{B}+\epsilon_{\mathbf{k} \uparrow}+\epsilon_{\mathbf{k} \downarrow}}\right. \\
& \left.+\frac{H\left(\left|\frac{\mathbf{p}}{2}+\mathbf{k}\right|-k_{F}\right)}{i \Omega-\epsilon_{\frac{\mathbf{p}}{2}-\mathbf{k} \downarrow}-\epsilon_{\frac{\mathbf{p}}{2}+\mathbf{k} \uparrow}+\mu+\varepsilon_{F}}\right] .
\end{aligned}
$$

The relevant parameter that characterizes the interaction in Eq. (6) is $\epsilon_{B}$. Equation (6) is well defined in the thermodynamic and $b \rightarrow 0$ limits. One finds

$$
\frac{1}{\Gamma^{0}(p, i \Omega)}=\frac{m}{4 \pi} \ln \left[\frac{2 \varepsilon_{B}}{-z+\sqrt{\left(z-\epsilon_{\mathbf{p}}\right)^{2}-4 \varepsilon_{F} \epsilon_{\mathbf{p}}}}\right],
$$

with $z \equiv i \Omega+\mu-\varepsilon_{F}$. In deriving the above expression for $\Gamma^{0}(p, i \Omega)$ we have taken $\mu<-\varepsilon_{F}$. Since Feynman diagrams for the self-energy will be evaluated in the momentumimaginary-time representation $(p, \tau)$, we need to evaluate the Fourier transform

$$
\Gamma^{0}(p, \tau)=\frac{1}{2 \pi} \int_{-\infty}^{+\infty} d \Omega e^{-i \Omega \tau} \Gamma^{0}(p, i \Omega) .
$$

In order to determine the leading behavior of $\Gamma^{0}(p, \tau)$ for small $\tau$, we introduce the vertex function $\tilde{\Gamma}^{0}$, which differs from $\Gamma^{0}$ by ignoring the Fermi surface when integrating out the internal momenta. This amounts to ignoring the Heaviside function in Eq. (5). We obtain

$$
\frac{1}{\tilde{\Gamma}^{0}(p, i \Omega)}=\frac{m}{4 \pi} \ln \left[-\frac{\varepsilon_{B}}{i \Omega+\mu+\varepsilon_{F}-\frac{\epsilon_{\mathrm{p}}}{2}}\right] .
$$

In the $(p, i \Omega)$ representation,

$$
\frac{1}{\Gamma^{0}}-\frac{1}{\tilde{\Gamma}^{0}}=\frac{m}{4 \pi} \ln \left[\frac{-2\left(z+2 \varepsilon_{F}\right)+\epsilon_{\mathbf{p}}}{-z+\sqrt{\left(z-\epsilon_{\mathbf{p}}\right)^{2}-4 \varepsilon_{F} \epsilon_{\mathbf{p}}}}\right] .
$$

The $(p, \tau)$ representation of $\tilde{\Gamma}^{0}$ is

$$
\begin{aligned}
\tilde{\Gamma}^{0}(p, \tau)= & -\frac{4 \pi \varepsilon_{B}}{m} e^{-\left(\frac{\epsilon_{\mathbf{p}}}{2}-\varepsilon_{F}-\mu\right) \tau}\left[\int_{0}^{+\infty} d x \frac{e^{-x \varepsilon_{B} \tau}}{\pi^{2}+\ln ^{2}(x)}\right. \\
& \left.+e^{\varepsilon_{B} \tau} H\left(\frac{\epsilon_{\mathbf{p}}}{2}-\varepsilon_{F}-\varepsilon_{B}-\mu\right)\right] H(\tau)
\end{aligned}
$$

for $\mu<-\varepsilon_{F}-\varepsilon_{B}$, ensuring that only $\tau>0$ contributes for all momenta $p$. The integral in Eq. (11) can be computed numerically but converges poorly for $\tau \rightarrow 0^{+}$. Under those conditions we make use of the asymptotic behavior:

$$
\int_{0}^{+\infty} d x \frac{e^{-x \varepsilon_{B} \tau}}{\pi^{2}+\ln ^{2}(x)} \underset{\tau \rightarrow 0}{\sim} \frac{1}{\varepsilon_{B} \tau} \frac{1}{\ln ^{2}\left(\varepsilon_{B} \tau\right)} .
$$

To obtain $\Gamma^{0}(p, \tau)$ we computed numerically the following Fourier transform:

$$
\begin{aligned}
\Gamma^{0}(p, \tau)-\tilde{\Gamma}^{0}(p, \tau)= & \frac{1}{2 \pi} \int_{-\infty}^{+\infty} d \Omega e^{-i \Omega \tau} \\
& \times\left[\Gamma^{0}(p, i \Omega)-\tilde{\Gamma}^{0}(p, i \Omega)\right] .
\end{aligned}
$$

The left-hand side of Eq. (13) can be computed more easily than $\tilde{\Gamma}^{0}(p, \tau)$ as it contains no singularities. Next, the function $\Gamma^{0}(p, \tau)$ is obtained as $\tilde{\Gamma}^{0}(p, \tau)+\left[\Gamma^{0}(p, \tau)-\tilde{\Gamma}^{0}(p, \tau)\right]$. Although the functions $\tilde{\Gamma}^{0}(p, \tau)$ and $\Gamma^{0}(p, \tau)$ are extremely sharp and divergent for $\tau \rightarrow 0$, they are integrable. Special care should be taken when using these functions in the Monte Carlo code. It is important to correctly sample very short times, and one needs to make sure there is no loss of accuracy when keeping track of imaginary time differences of the $\Gamma^{0}$ lines in the diagrams. Just like for the 3D polaron problem [14-16], we consider a diagrammatic series for the self-energy built from the free one-body propagators for the impurity and the spin-up Fermi sea and from the renormalized interaction $\Gamma^{0}$. We refer to this series as the bare series, which we evaluate 


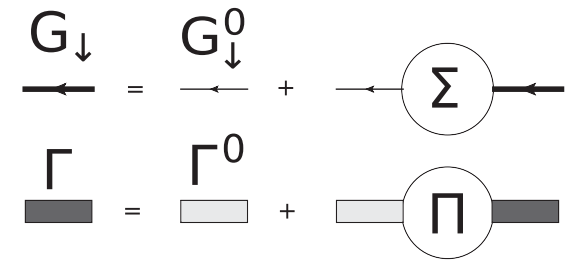

FIG. 1. Graphical representation of the Dyson equation. The free (dressed) one-body impurity propagator is denoted by $G_{\downarrow}^{0}\left(G_{\downarrow}\right)$. $\Sigma$ and $\Pi$ are the one-body and two-body self-energies, respectively. $\Gamma$ is the fully dressed interaction, whereas $\Gamma^{0}$ is the partially dressed interaction as shown in Eq. (3).

with the DiagMC method. The diagram topologies in 2D and $3 \mathrm{D}$ are exactly the same. The major differences between the diagrammatic-series evaluations in $2 \mathrm{D}$ and $3 \mathrm{D}$ are the renormalized interaction $\Gamma^{0}(p, \tau)$ and the phase-space volume elements. The one and two-body self-energies are related to the one-particle propagator $G$ and the fully dressed interaction $\Gamma$ by means of a Dyson equation, as illustrated schematically in Fig. 1. From the poles of $G$ and $\Gamma$ we can extract the polaron and the molecule energy, respectively. The fully dressed interaction is closely related to the two-particle propagator [16].

For the 3D Fermi polaron problem there are two dominant diagrams at each given order that emerge next to many diagrams with a much smaller contribution [16]. These dominant diagrams contribute almost equally but have opposite sign. In $2 \mathrm{D}$, however, the numerical calculations indicate that at a given order the very same two diagrams dominate, but to a lesser extent; that is, the nondominant diagrams have a larger weight in the final 2D result. By weight of a given diagram we mean the absolute value of its contribution to the self-energy. We note that the sign of a single diagram at fixed internal and external variables depends only on its topology and not on the values of the internal and external variables. We stress that this is not true for a Fermi system with two interacting components with finite density $[27,28]$. In 2D the total weight of a given order (i.e., the sum of the absolute values of the contributions of diagrams) is distributed over more diagrams than in 3D. Because the sign alternation occurs over a broader distribution of the weights, we get more statistical noise in sampling the self-energy in 2D compared to 3D. In 3D we can evaluate the diagrammatic series for the one-body self-energy accurately up to order 12, whereas in 2D we can reach order 8 .

In principle, other choices for the propagators ("bare" versus "dressed" propagators) are possible, and this was discussed in detail for the 3D Fermi polaron in our previous paper [16]. Replacing the bare propagators by dressed ones reduces the number of diagrams at each given order. One may expect that this replacement could allow one to reach higher orders. For the 3D polaron, however, the most favorable conditions of cancellations between the contributions from the various diagrams were met in the bare scheme [16]. In the DiagMC framework a higher accuracy can be reached under conditions of strong cancellations between the various contributions. From numerical investigations with various propagators for the 2D Fermi polaron we could draw similar conclusions as in the 3D studies. Accordingly, all numerical results for the quasiparticle properties presented below are obtained for a series expansion with bare propagators.

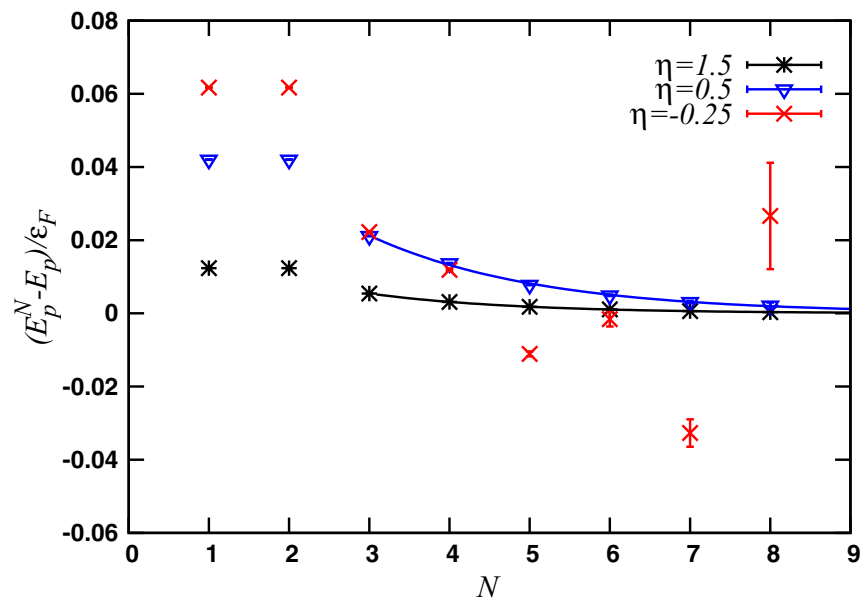

FIG. 2. (Color online) Dependence of the polaron energy $E_{p}^{N}$ on the cutoff diagram order $N . E_{p}$ is the value obtained after extrapolation to $N \rightarrow+\infty$ (and resummation for $\eta=-0.25$ ). Results are shown for $\eta=-0.25, \eta=0.5, \eta=1.5$. The lines represent an exponential fit.

To characterize the magnitude of the interaction strength we use the dimensionless parameter $\eta \equiv \ln \left[k_{F} a_{2 D}\right]=$ $\ln \left[2 \varepsilon_{F} / \varepsilon_{B}\right] / 2$. Here, $a_{2 D}>0$ is the $2 \mathrm{D}$ scattering length, related to the dimer binding energy by $\varepsilon_{B}=1 /\left(2 m_{r} a_{2 D}^{2}\right)$ with $m_{r}=m_{\uparrow} m_{\downarrow} /\left(m_{\uparrow}+m_{\downarrow}\right)$ being the reduced mass. The BCS regime corresponds to $\eta \gg 1$ while the Bose-Einstein condensate (BEC) regime corresponds to $\eta \ll-1$. The system is perturbative in the regimes $|\eta| \gg 1$, while the strongly correlated regime corresponds to $|\eta| \lesssim 1$ [29]. In the weakcoupling regime [small interaction strengths $g_{0}$ in the Hamiltonian of Eq. (1) or large positive $\eta$ in the zero-range limit], we find that the one-body and the two-body self-energy $\Sigma$ and $\Pi$ converge absolutely as a function of the maximum diagram order. This is demonstrated in Fig. 2 for $\eta=1.5$, where the polaron energy $E_{p}^{N}$ converges exponentially as a function of the cutoff diagram order $N$. Similar convergence is also found for the molecule energy. Under conditions of convergence with diagram order, extrapolation to order infinity can be done in a trivial way. Similar convergence is also seen for $\eta=0.5$. In the strongly correlated regime the series starts oscillating with order for the situation $\eta \lesssim 0$, and the oscillations get stronger the deeper we go into the BEC regime. The oscillations in the extracted polaron energy are illustrated in Fig. 2 for $\eta=-0.25$. To obtain meaningful results we rely on Abelian resummation techniques $[16,27]$. We evaluate the series $\sigma_{\epsilon}=\sum_{N} \sigma^{(N)} e^{-\epsilon \lambda_{N}}$, with $\sigma^{N}$ being the one-body self-energy for diagram order $N$ and $\lambda_{N}$ being a function that depends on the diagram order $N$. For each $\epsilon$ the polaron energy $E_{p}$ is calculated from $\sigma_{\epsilon}$, and an extrapolation is done by taking the limit $\epsilon \rightarrow 0$. The whole procedure is illustrated in Fig. 3. To estimate the systematic error of the extrapolation procedure, different resummation functions $\lambda_{N}$ are used. As becomes clear from Fig. 3 the whole resummation procedure is a stable one and induces uncertainties on the extracted energies of the order of a few percent. All the results of Fig. 4 are obtained with the Abelian resummation technique. The stronger the coupling constant is, 


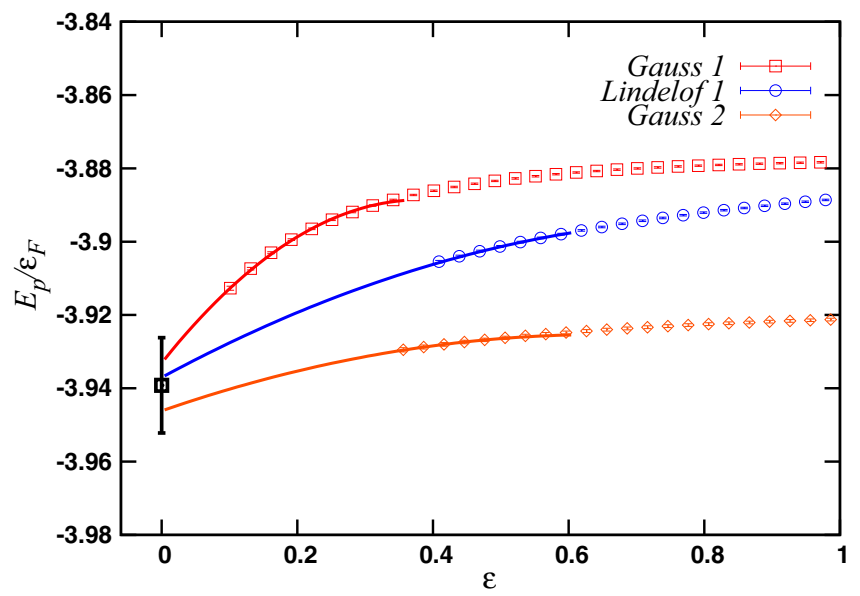

FIG. 3. (Color online) Abelian resummation of the bare series for the one-body self-energy diagrams at $\eta=-0.25$. We evaluate $\sigma_{\epsilon}=\sum_{N} \sigma^{(N)} e^{-\epsilon \lambda_{N}}$, with $\sigma^{N}$ being the one-body self-energy for diagram order $N$. We use the following functions $\lambda_{N}$ : (i) Gauss 1: $\lambda_{N}=(N-1)^{2}$ for $N>1$ and $\lambda_{N}=0$ for $N=1$, (ii) Lindelöf 1 : $\lambda_{N}=(N-1) \log (N-1)$ for $N>2$ and $\lambda_{N}=0$ for $N \leqslant 2$, and (iii) Gauss 2: $\lambda_{N}=(N-3)^{2}$ for $N>3$ and $\lambda_{N}=0$ for $N \leqslant 3$. The polaron energy $E_{p} / \epsilon_{F}$ is extracted in the limit $\epsilon=0^{+}$for various choices of $\lambda_{N}$.

the larger the size of the error attributed to the resummation is. An accurate extrapolation to infinite diagram order could be achieved for all values of $\eta$.

\section{RESULTS AND DISCUSSION}

In Fig. 4, polaron and molecule energies are displayed for a wide range of the parameter $\eta$. DiagMC results include all diagrams up to order 8 and extrapolation to the infinite diagram order. In the region $\eta \lesssim 0$ a small discrepancy (of the order of

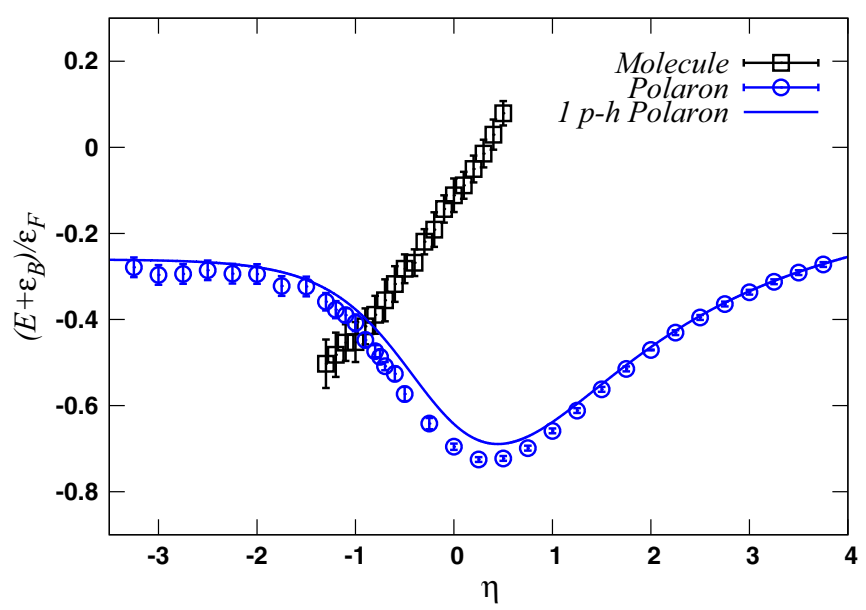

FIG. 4. (Color online) Polaron and molecule ground-state energies $E$ in units of the Fermi energy $\varepsilon_{F}$ as a function of $\eta$. The momentum of the impurity is equal to zero. Energies are shifted by the two-body binding energy $\varepsilon_{B} / \varepsilon_{F}=2 e^{-2 \eta}$ to magnify the details. The solid line is the DiagMC result for $N=1$. The symbols are the result of the full DiagMC calculations (including diagrams up to order 8).
$0.1 \%$ of the ground-state energy) is found with the variational results [25] of Parish and Levinsen based on the wave-function Ansatz up to $2 \mathrm{p}-\mathrm{h}$ excitations. Clearly, a phase transition appears at the critical value $\eta_{c}=-0.95 \pm 0.15$. A variational result which includes $2 \mathrm{p}-\mathrm{h}$ excitations for the polaron and 1p-h excitations for the molecule gives $\eta=-0.97$ [25]. Both mentioned calculations are in agreement with the experimental result $\eta=-0.88(0.20)$ [11].

The DiagMC method allows one to include a large number of particle-hole excitations that dress the impurity. Truncation of the Hilbert space to a maximum number of $\mathrm{p}$-h pairs can nonetheless be achieved within the DiagMC approach. This allows one to arrive at the variational formulation. Previous variational studies using a wave function Ansatz up to $1 \mathrm{p}-\mathrm{h}$ or $2 \mathrm{p}$-h excitations showed that these truncations give remarkably accurate results [30].

To understand why the truncation is possible within a Feynman diagrammatic approach for the self-energy, we first remark that a variational approach is easily established within a path-integral formalism. Path integrals with continuous imaginary time, for example, are based on an expansion of the evolution operator,

$$
\begin{aligned}
e^{-\beta \hat{K}}= & e^{-\beta \hat{K}_{0}}\left(1-\int_{0}^{\beta} d \tau_{1} K_{1}\left(\tau_{1}\right)\right. \\
& \left.+\int_{0}^{\beta} d \tau_{1} \int_{0}^{\tau_{1}} d \tau_{2} \hat{K}_{1}\left(\tau_{1}\right) \hat{K}_{1}\left(\tau_{2}\right)-\cdots\right),
\end{aligned}
$$

where $\hat{K}=\hat{H}-\mu \hat{N}=\hat{K}_{0}+\hat{K}_{1}-\mu \hat{N}$, with $\left[\hat{K}_{0}, \hat{K}_{1}\right] \neq 0$. The operator $\hat{K}_{1}(\tau)=e^{\hat{K}_{0} \tau} \hat{K}_{1} e^{-\hat{K}_{0} \tau}$, which defines the series expansion, is expressed in the interaction picture. Further, $\beta=1 / k_{B} T$, with $k_{B}$ being Boltzmann's constant and $T$ being temperature, $\hat{H}$ is the Hamiltonian, $\hat{N}$ is the number operator, and $\mu$ is the chemical potential. The imaginary-time evolution operator in Eq. (14) can be used as a ground-state projection operator: for sufficiently long imaginary time $\beta$ the excitedstate components of a trial state are exponentially suppressed. One typically evaluates all the terms in the expansion (14) in the eigenbasis of $\hat{K}_{0}$. This procedure forms the basis of path-integral Monte Carlo simulation of lattice models, where $\hat{K}_{1}$ is usually the kinetic energy term [31]. A discretized time version is used in path-integral Monte Carlo methods in continuous space $[32,33]$. Either way, the contributions to the path integral have the direct physical interpretation of a time history of the many-particle system. At each instant of time, one can constrain the accessible states of the Hilbert space, in line with what is done in a variational approach. Within the standard Feynman diagrammatic formalism for Green's functions, however, this truncation of the Hilbert space is not easy to accomplish for an arbitrary system, as one expands in powers of the two-body interaction term of the Hamiltonian. This will be explained in the next paragraph.

It turns out to be formally easier to start from finite $T$ and to take the $\beta \rightarrow \infty$ limit in the end. For a many-fermion system, the finite-temperature Green's function in position and imaginary-time representation $(\mathbf{x}, \tau)$ is defined as

$$
G_{\alpha \sigma}(\mathbf{x}, \tau)=-\frac{\operatorname{Tr}\left[e^{-\beta \hat{K}} T_{\tau} \hat{\psi}_{H \alpha}(\mathbf{x}, \tau) \hat{\psi}_{H \sigma}^{\dagger}(\mathbf{x}, 0)\right]}{\operatorname{Tr}\left[e^{-\beta \hat{K}}\right]},
$$


with $\alpha$ and $\sigma$ denoting an appropriate set of quantum numbers (such as spin) and $T_{\tau}$ being the time-ordering operator. The field operator in the Heisenberg picture $\hat{\psi}_{H \alpha}(\mathbf{x}, \tau)=$ $e^{\hat{K} \tau} \hat{\psi}_{\alpha}(\mathbf{x}) e^{-\hat{K} \tau}$ annihilates a fermion in state $\alpha$ at position $\mathbf{x}$ and time $\tau$. To arrive at the Feynman diagrammatic expansion, one makes a perturbative expansion for the evolution operator $e^{-\beta \hat{K}}$ in both the numerator and the denominator of Eq. (15) (the finite $T$ ensures that both exist). The expansion of the partition function $Z$ in the denominator can be represented graphically by the series of all fully closed diagrams (connected and disconnected). When $\beta$ approaches $+\infty$, the denominator is proportional to $\left\langle\Psi_{0} \mid \Psi_{0}\right\rangle \quad\left(\left|\Psi_{0}\right\rangle\right.$ is the ground state of the interacting many-body system), and the disconnected diagrams correspond to all possible vacuum fluctuations of the system at hand. The expansion in the numerator factorizes into an expansion of connected diagrams for $G_{\alpha \sigma}$ and disconnected diagrams for $Z$. So the sum of disconnected diagrams drops out, as expected for an intensive quantity like $G_{\alpha \sigma}(\mathbf{x}, \tau)$. It is exactly this factorization that prevents one from truncating the Hilbert space at any instant of time in the evolution. In other words, variational calculations based on Feynman diagrams for the self-energy are generally not feasible.

In the polaron problem vacuum fluctuations are absent since $\left|\Psi_{0}\right\rangle$ corresponds to the spin-down vacuum and a noninteracting spin-up Fermi sea. In other words, the vacuum cannot be polarized in the absence of an impurity. As a consequence, we face a situation similar to the path integral with a direct physical interpretation of the time history of the impurity. This peculiar feature allows one to restrict the Hilbert space at each given time. If we allow at most $1 \mathrm{p}-\mathrm{h}$ excitations at each instant of time, only one diagram survives: the lowest-order self-energy diagram built from $\Gamma^{0}$ and the free spin-up single-particle propagator $G_{\uparrow}^{0}$. The equivalence between this diagram and the $1 \mathrm{p}-\mathrm{h}$ variational approach had already been pointed out in Ref. [21]. An $n \mathrm{p}-\mathrm{h}$ variational approach is achieved by allowing at most $n$ backward spin-up lines at each step in the imaginary-time evolution.

For large $\eta$ it is obvious from Fig. 4 that the polaron energy from the full series expansion becomes equal to the $1 \mathrm{p}$-h result. Even for stronger interactions (smaller $\eta$ ) the first-order results remain close to the full DiagMC one. Within the statistical accuracy of the numerical calculations, convergence for the one-body self-energy is already reached after inclusion of $2 \mathrm{p}-\mathrm{h}$ excitations. Indeed, for all values of $\eta$, we find agreement between our $2 p-h$ variational DiagMC approach and the full DiagMC approach within statistical error bars. For the molecular branch, we retrieve the result for the two-body self-energy from the full series expansion after including $1 \mathrm{p}$-h excitations. For the 3D Fermi-polaron a similar conclusion was drawn. Also in 3D, the first-order result is a very good approximation [16]. Going up to $2 \mathrm{p}$-h pairs gives a perfect agreement with full DiagMC results. From the above considerations it follows, however, that the diagrammatic truncations which provide good results for the polaron problem may not be appropriate for the more complex many-body problem with comparable densities for both components.

The quasiparticle residue or $Z$ factor of the polaron gives the overlap of the noninteracting wave function and the fully interacting one. This overlap is very small for a molecular

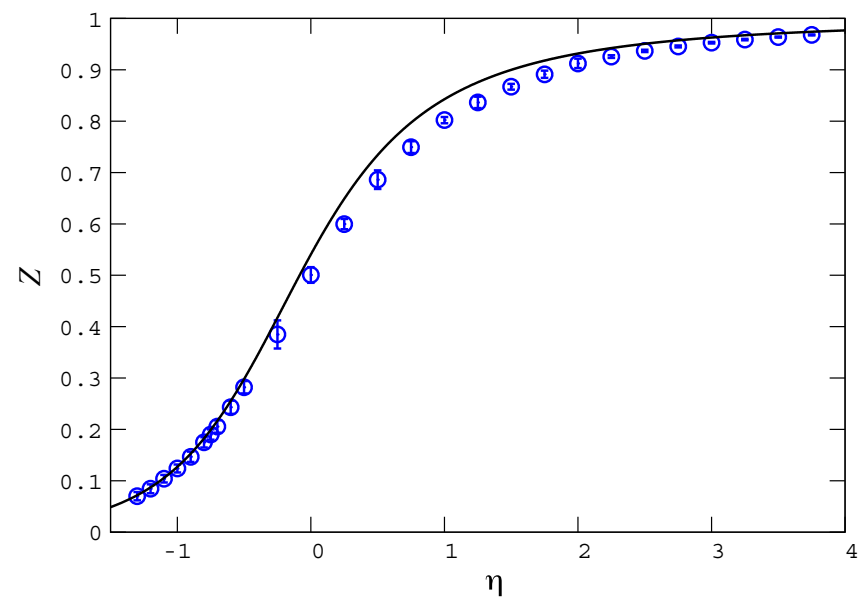

FIG. 5. (Color online) The quasiparticle residue $Z$ of the polaron as a function of $\eta$. The solid line represents the $1 \mathrm{p}-\mathrm{h}$ result $(N=1$ diagram).

ground state of the fully interacting system [16]. The $Z$ factor as a function of $\eta$ is shown in Fig. 5. Note that the polaron $Z$ factor does not vanish in the region $\eta \lesssim-1$ where the ground state is a molecule. The $Z$ factor is, however, still meaningful since the polaron is a well-defined (metastable) excited state of the 2D system. Again, the first-order result gives a good approximation to the full result. The measured $Z$ factor for the 3D situation has been reported in Ref. [10]. The 2D experimental data are reported in Ref. [11], and the $\eta$ dependence of the quasiparticle weight $Z$ is presented in arbitrary units. We reproduce the observation that $Z$ strongly increases between $\eta_{c} \lesssim \eta \lesssim 1$ and saturates to a certain value for $\eta>1$.

\section{CONCLUSION}

Summarizing, we have developed a framework to study with the DiagMC method the ground-state properties of the 2D Fermi polaron for attractive interactions. We have shown that the framework allows one to select an arbitrary number of $n \mathrm{p}$-h excitations of the FS, thereby making a connection with typical variational approaches which are confined to $n=1$ and $n=2$. We have studied the quasiparticle properties of the ground state for a wide range of interaction strengths. A phase transition between the polaron and molecule states is found at interaction strengths compatible with experimental values and with variational predictions. To a remarkable degree, it is observed that for all interaction strengths the full DiagMC results (which include all $n \mathrm{p}$-h excitations) for the ground-state properties can be reasonably approximated by $n=1$ truncations. In an $n=2$ truncation scheme the full result is already reached within the error bars. This lends support for variational approaches to the low-dimensional polaron problem, for which one could have naively expected a large sensitivity to quantum fluctuations.

\section{ACKNOWLEDGMENTS}

This work is supported by the Fund for Scientific researchFlanders. We would like to thank C. Lobo, N. Prokof'ev, B. Svistunov, and F. Werner for helpful discussions and suggestions, and we thank J. Levinsen and M. Parish for sending us their data. 
[1] C. Chin, R. Grimm, P. Julienne, and E. Tiesinga, Rev. Mod. Phys. 82, 1225 (2010).

[2] I. Bloch, Nat. Phys. 1, 23 (2005).

[3] G. B. Partridge, W. Li, Y. A. Liao, R. G. Hulet, M. Haque, and H. T. C. Stoof, Phys. Rev. Lett. 97, 190407 (2006).

[4] Y. Shin, C. H. Schunck, A. Schirotzek, and W. Ketterle, Nature (London) 451, 689 (2008).

[5] S. Nascimbène, N. Navon, K. J. Jiang, L. Tarruell, M. Teichmann, J. McKeever, F. Chevy, and C. Salomon, Phys. Rev. Lett. 103, 170402 (2009).

[6] S. Pilati and S. Giorgini, Phys. Rev. Lett. 100, 030401 (2008).

[7] P. Massignan, Z. Yu, and G. M. Bruun, Phys. Rev. Lett. 110, 230401 (2013).

[8] G. Bertaina and S. Giorgini, Phys. Rev. Lett. 106, 110403 (2011).

[9] F. Chevy, Phys. Rev. A 74, 063628 (2006).

[10] A. Schirotzek, C.-H. Wu, A. Sommer, and M. W. Zwierlein, Phys. Rev. Lett. 102, 230402 (2009).

[11] M. Koschorreck, D. Pertot, E. Vogt, B. Fröhlich, M. Feld, and M. Köhl, Nature (London) 485, 619 (2012).

[12] P. Dyke, E. D. Kuhnle, S. Whitlock, H. Hu, M. Mark, S. Hoinka, M. Lingham, P. Hannaford, and C. J. Vale, Phys. Rev. Lett. 106, 105304 (2011).

[13] J. Levinsen and S. K. Baur, Phys. Rev. A 86, 041602 (2012).

[14] N. V. Prokof'ev and B. V. Svistunov, Phys. Rev. B 77, 020408 (2008).

[15] N. V. Prokof'ev and B. V. Svistunov, Phys. Rev. B 77, 125101 (2008).

[16] J. Vlietinck, J. Ryckebusch, and K. Van Houcke, Phys. Rev. B 87, 115133 (2013).
[17] M. Punk, P. T. Dumitrescu, and W. Zwerger, Phys. Rev. A 80, 053605 (2009).

[18] R. Combescot, S. Giraud, and X. Leyronas, Europhys. Lett. 88, 60007 (2009).

[19] C. Mora and F. Chevy, Phys. Rev. A 80, 033607 (2009).

[20] J. B. McGuire, J. Math. Phys. 7, 123 (1966).

[21] R. Combescot, A. Recati, C. Lobo, and F. Chevy, Phys. Rev. Lett. 98, 180402 (2007).

[22] S. Giraud and R. Combescot, Phys. Rev. A 79, 043615 (2009).

[23] S. Zöllner, G. M. Bruun, and C. J. Pethick, Phys. Rev. A 83, 021603(R) (2011).

[24] M. M. Parish, Phys. Rev. A 83, 051603(R) (2011).

[25] M. M. Parish and J. Levinsen, Phys. Rev. A 87, 033616 (2013).

[26] L. D. Landau and E. M. Lifshitz, Statistical Physics (Pergamon, New York, 1980), Vol. 2.

[27] K. Van Houcke, F. Werner, E. Kozik, N. Prokof'ev, B. Svistunov, M. J. H. Ku, A. T. Sommer, L. W. Cheuk, A. Schirotzek, and M. W. Zwierlein, Nat. Phys. 8, 366 (2012).

[28] K. Van Houcke, F. Werner, N. Prokof'ev, and B. Svistunov, arXiv:1305.3901.

[29] P. Bloom, Phys. Rev. B 12, 125 (1975).

[30] R. Combescot and S. Giraud, Phys. Rev. Lett. 101, 050404 (2008).

[31] N. V. Prokof'ev, B. V. Svistunov, and I. S. Tupitsyn, J. Exp. Theor. Phys. 87, 310 (1998).

[32] D. M. Ceperley, Rev. Mod. Phys. 67, 279 (1995).

[33] M. Boninsegni, Nikolay Prokof'ev, and B. Svistunov, Phys. Rev. Lett. 96, 070601 (2006). 